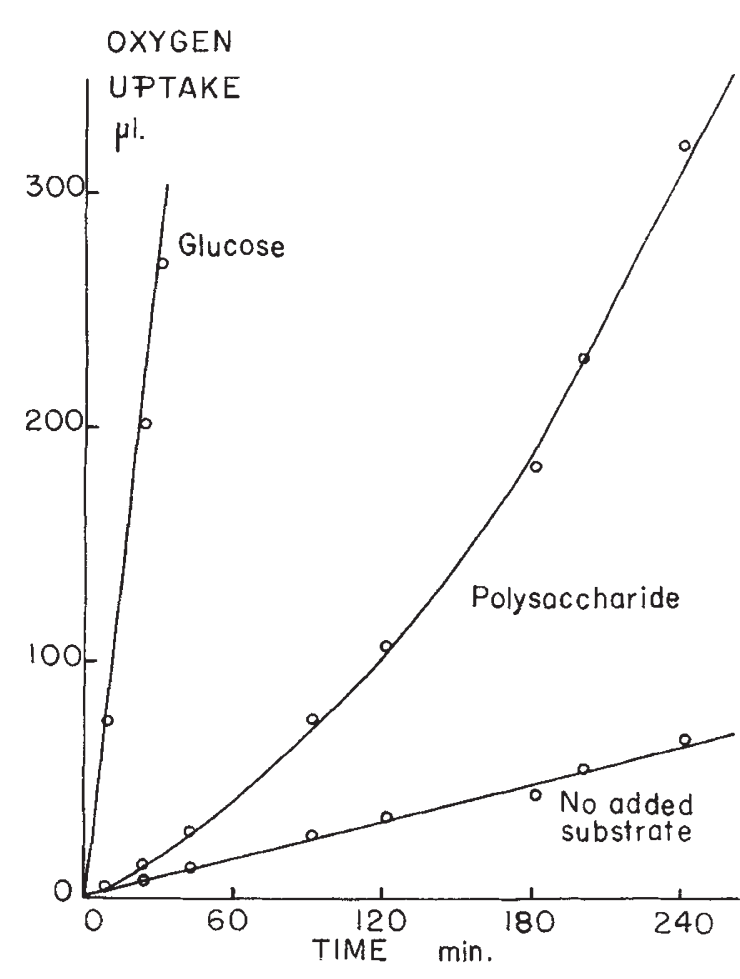

Fig. 2. The oxidation of polysaccharide by Azotobacter vinelandi in early growth on ethanol medium (stage $(a)$ )

Nitrogen-free mineral salts medium solidified with agar was prepared: without added carbon source; with a trace of acetone as carbon source; with ethanol as carbon source; and with polysaccharide as carbon source. Periodic transfers were made from the ethanol medium to these media with the following results. Cells from stage $(a)$ grew rapidly on ethanol, and after a lag period of approximately $24 \mathrm{hr}$. on the polysaccharide medium. Cells from stages $(c)$ and $(d)$ grew rapidly and luxuriantly on both ethanol and polysaccharide, and produced clearing of the initially opaque polysaccharide medium. Cells from any growth stage grew to a negligible extent on the control media.

Oxidation of the polysaccharide was studied with standard manometric technique. After harvest from the surface of the ethanol medium, the cells were washed by centrifugation and resuspended in $0.01 M$ phosphate buffer $p \mathrm{H} 7 \cdot 0$, prior to aeration before use. The oxygen uptake from a gas phase of air was observed with polysaccharide as substrate. Controls with glucose were included to indicate the general physiological state of the cells. The results in Fig. 1 show that the organisms in stage $(d)$ are able to metabolize the polysaccharide without lag at a rate significantly above the endogenous; while the results in Fig. 2 show that organisms in stage $(a)$ are also able to metabolize the polysaccharide, but only after a lag period. Cultures grown with polysaccharide as carbon source metabolize as stage $(d)$ organisms.

All these observations are consistent with the postulate that the organisms are able to use their extra-cellular polysaccharide as a carbon and energy source. Thus the polysaccharide may be regarded. as a true storage material.
These experiments were carried out at the Hopkins Marine Station, Pacific Grove, California, and I would like to thank Dr. C. B. van Niel for the hospitality of his laboratory.

Department of Biochemistry, Michael H. Proctor

University of Cambridge. Aug. 22.

2 Proctor, M. H., and Wilson, P. W., Nature, 182, 891 (1958).

${ }^{2}$ Proctor, M. H., and Wilson, P. W., Bact. Proc., 112 (1959)

${ }^{3}$ Beijerinck, M. W., and den Dooren de Jong, L. E., Kon. Akad. van Wetenschappen A musterdam, Proc, 25, 279 (1922)

4 Proctor, M. H., and Wilson, P. W. (in preparation). Breed, R. S., Murray, E. G. D., and Hitchens, A. P.' 'Bergey' Wilkins. Co., Baltimore, 1948).

\section{Occurrence of Leuco-Anthocyanin in Sour and Sweet Cherries}

In recent years there has been an increasing interest in the role of leuco-anthocyanins as possible precursors of anthocyanins in vivo. Evidence for and against this hypothesis has been presented by many workers, and reviewed by Alston ${ }^{1}$. In the course of the isolation and identification of the anthocyanin pigments of sour cherries $^{2}$ and sweet cherries ${ }^{3}$ in this Laboratory, these fruits at two stages of maturity were examined for the occurrence of leuco-anthocyanins. Evidence was found for the presence of the leuco-anthocyanin related to cyanidin, which is also the single nucleus for the anthocyanin pigments in cherries ${ }^{2,3}$.

Green and yellow fruits of sour cherry (Prunus cerasus L., var. Montmorency) and two varieties of sweet cherry (Prunus avium L.), the Windsor and Yellow Glass, were found to have leuco-anthocyanins in both the peels and flesh by a method 4 of detection involving heating the plant tissues in $2 \mathrm{~N}$ hydroehloric acid at the temperature of boiling water for $20 \mathrm{~min}$., and extracting the red anthocyanidin pigment so formed with iso-amyl alcohol. This derived pigment was shown to consist solely of cyanidin in all cases by paper chromatography, colour reactions and absorption spectra, in comparison with authentic cyanidin, as previously described ${ }^{2,3}$.

It is to be noted that the amount of cyanidin, derived from the leuco-anthocyanin, was 5-6 times as much as obtained from sour cherries, than from sweet cherries at a similar stage of maturity. The yield of cyanidin from the two varieties of sweet cherries was about the same. The concentration of cyanidin in the acidified iso-amyl alcohol solution was estimated by the optical density of absorption at $550 \mathrm{~m} \mu$. As the cherry fruits matured, such amounts of cyanidin decreased in all cases. However, an interesting fact is that the colour of the fruits of the Yellow Glass variety of sweet eherry, unlike the Windsor variety, is yellow though fully ripened.

\section{KuANG C. LI*}

New York State Agricultural Experiment Station, Cornell University, Geneva,

New York. Sept. 11.

* Present address : Citrus Experiment Station, University of Florida, Lake Alfred, Florida.

1 Alston, R. E., Amer. J. Bot., 45, 289 (1958).

$2 \mathrm{Li}, \mathrm{K} . \mathrm{C}$., and Wagenknecht, A. C., J. Amer. Chem. Soc., 78, 979 (1956). ${ }^{8} \mathrm{Li}, \mathrm{K}$. C., and Wagneknecht, A. C., Nature, 182, 657 (1958).

4 Bate-Smith, E. C., Biochem. J., 58, 122 (1954). 